\title{
Kinematics of Yang-Mills Solitons
}

\author{
John L. Friedman ${ }^{1, \star}$ and Rafael D. Sorkin ${ }^{2}$ \\ 1 Physics Department, University of Wisconsin-Milwaukee, Milwaukee, WI 53201, USA \\ 2 Institute for Advanced Study, Princeton, NJ 08540, and Center for Theoretical Physics, \\ University of Maryland, College Park, MD 20742, USA
}

\begin{abstract}
In SU(2) Yang-Mills theory, the $N$-monopole configuration space is a bundle with fibers isomorphic to $\mathrm{U}(1) \times \ldots \times \mathrm{U}(1)$, and state vectors for which each monopole has charge $n \frac{e}{2}$ are homogeneous of degree $n$ with respect to each U(1). Translations and rotations are defined for individual monopoles in the $N$-monopole space. The commutator of two translations is found to be a U(1) transformation that agrees for large monopole separation with the analogous phase change accompanying the translation of a charged point particle in an external magnetic field. The theory developed here is applied in a companion paper to prove a spin-statistics theorem for monopoles in SU(2) Yang-Mills theory.
\end{abstract}

\section{Introduction}

We examine here the kinematics of non-overlapping monopoles in an SU(2) YangMills-Higgs theory [1]. In Sect. II we briefly review the quantum theory of YangMills monopoles in the Schrödinger representation $[2,3]$. We observe first that for a single monopole, the configuration space $\mathscr{C}$ on which the state functional $\psi$ takes its values is a principle $\mathrm{U}(1)$ bundle; and state vectors of charge $Q_{E}=n \frac{e}{2}$ are homogeneous functionals of degree $n$ on the bundle, where $\frac{e}{2}$ is the smallest unit of electric charge. We define rotations and translations in the one-monopole sector by requiring that these transformations leave unchaged the asymptotic behaviour of the fields. It then follows that the state space includes states for which the corresponding angular momentum operators have half integral values.

In analyzing monopole kinematics, our principal aim is to define translations and rotations of individual monopoles in the presence of other monopoles, and

* Supported in part by the National Science Foundation 
this is carried out in Sect. III. We first introduce a configuration space $\mathscr{C}_{m}^{N}$ of $N$ distinct monopoles each with magnetic charge $Q_{B}=-\frac{m}{e}$. The space $\mathscr{C}_{m}^{N}$ is again a bundle with a $U(1)$ degree of freedom for each monopole - a bundle with fibers isomorphic to the product $\mathrm{U}(1) \times \ldots \times \mathrm{U}(1)$; and state vectors for which each monopole has electric charge $Q_{E}$ are homogeneous of degree $n$ with respect to each $\mathrm{U}(1)$ in the product.

A decomposition of the fields inside a monopole into an "internal configuration" and a magnetic Coulomb field due to the other monopoles is then introduced. This decomposition (which at large monopole separation is physically appropriate) leads to the desired definitions of individual monopole translation and rotation. An intermediate step, and a possibly useful byproduct of the work, is a definition of monopole translation and rotation in an external field.

\section{Preliminaries}

We consider a theory of an SU(2) Yang-Mills field minimally coupled to two scalar fields, one a Higgs field $\Phi^{a}$ in the adjoint (isovector) representation and the other a spinless isodoublet field $\sigma^{I}$. The Lagrangian density will be

$$
\begin{aligned}
\mathscr{L}= & -\frac{1}{16 \pi e^{2}} F_{\mu \nu}^{a} F_{a}{ }^{\mu \nu}+\frac{\theta}{32 \pi^{2}} F_{\mu \nu}^{a}{ }^{*} F_{a}{ }^{\mu \nu}-\frac{c^{2}}{2} D_{\mu} \Phi_{a} D^{\mu} \Phi^{a}-D_{\mu} \sigma_{I}^{\dagger} D^{\mu} \sigma^{I} \\
& -V\left(\Phi^{a} \Phi_{a}, \sigma_{I}^{\dagger} \sigma^{I}\right),
\end{aligned}
$$

where

$$
F_{\mu \nu}^{a}=\partial_{\mu} A^{a}{ }_{\nu}-\partial_{\nu} A^{a}{ }_{\mu}+\varepsilon^{a}{ }_{b c} A^{b}{ }_{\mu} A^{c}{ }_{\nu} .
$$

Here $\mu, v, \ldots$ are spacetime indices, $a, b, \ldots$ are Lie algebra $\left(\mathrm{So}_{3}\right)$ indices, and $I, J, \ldots$ are indices in the space of the isodoublet (two component isospinor) field $\sigma^{I}$. Latin indices $i, j, \ldots$ will denote spatial vectors. By $D_{\mu} \Phi^{a}$ and $D_{\mu} \sigma^{I}$ are meant the gauge covariant derivatives of the Higgs field,

$$
D_{\mu} \Phi^{a}=\partial_{\mu} \Phi^{a}+\varepsilon_{b c}^{a} A_{\mu}^{b} \Phi^{c}
$$

and of the isodoublet

$$
D_{\mu} \sigma^{I}=\partial_{\mu} \sigma^{I}-\frac{i}{2} \tau_{a J}^{I} A^{a}{ }_{\mu} \sigma^{J}
$$

We have normalized $A^{a}{ }_{\mu}$ so that no coupling constant occurs in (3) and (4) and $\Phi^{a}$, so that $\Phi^{a} \Phi_{a}=1$ in the vacuum. (This last feature can be obtained by choosing $V$ to be minimized at $|\Phi|=1$ or, à la Prasad-Sommerfield, simply by setting $V=0$ and demanding $|\Phi| \rightarrow 1$ as a boundary condition at spatial infinity.)

Finally, the term $\frac{\theta}{32 \pi^{2}} F_{\mu \nu}^{a}{ }^{*} F_{a}{ }^{\mu v}$ is a total divergence, which therefore does not alter the field equations. However, for Yang-Mills fields that interpolate between topologically distinct vacua related by a spatial gauge transformation of winding number $k$, the term gives a contribution to the action proportional to $k$ [4]. But 
the freedom to choose $\theta$ is redundant: it is equivalent to the freedom already present in the form of the superselected phase change $\hat{\theta}$ that results from a gauge transformation with nonzero winding number $[2,4]$. By including $\theta$ in the Lagrangian one can without loss of generality assume that $\hat{\theta}$ vanishes, and this will simplify the structure of the $N$-monopole configuration space. (We will indicate differences that arise when $\hat{\theta} \neq 0$.)

Denoting by $U^{I}{ }_{J}$ an element of SU(2) and by $R^{a}{ }_{b}$ the corresponding element of $\mathrm{SO}(3)$, we can write a gauge transformation of the three fields $\mathscr{F} \equiv(A, \Phi, \sigma)$ in the manner $\mathscr{F} \rightarrow \underset{\sim}{U F}$, which will mean

$$
\begin{gathered}
A_{\mu}^{a}{ }_{\mu} \rightarrow R_{b}^{a} A^{b}{ }_{\mu}+\varepsilon^{a b c} R_{d b} \partial_{\mu} R_{c}^{d}, \\
\sigma^{I} \rightarrow U^{I}{ }_{J} \sigma^{J}, \\
\Phi^{a} \rightarrow R^{a}{ }_{b} \Phi^{b} .
\end{gathered}
$$

An infinitesimal gauge transformation associated with the element $\varepsilon_{b c}^{a} \zeta^{c}$ of the Lie algebra will be written $\Delta\left(\zeta^{c}\right)$, where

$$
\begin{aligned}
\Delta A_{\mu}^{a} & :=D_{\mu} \zeta^{a}, \\
\Delta \Phi^{a}: & =\varepsilon_{b c}^{a}{ }_{b c} \zeta^{c} \Phi^{b}, \\
\Delta \sigma^{I}: & =\frac{i}{2} \tau_{a}{ }^{I} \zeta^{a} \sigma^{J} .
\end{aligned}
$$

It will also be useful to introduce an operator $\mathscr{D}_{\mu}$ whose action on $\mathscr{F}$ is [5]

$$
\mathscr{D}_{\mu} \mathscr{F}:=\left(F^{a}{ }_{\mu \nu}, D_{\mu} \Phi^{a}, D_{\mu} \sigma^{I}\right) \text {. }
$$

Then

$$
\xi^{\mu} \mathscr{D}_{\mu}=£_{\xi}-\Delta\left(A^{a}{ }_{\mu} \xi^{\mu}\right)
$$

where $£_{\xi}$ is the ("naive") Lie derivative with respect to $\xi^{\mu}$.

a. The Schrödinger Representation. We will adopt the Schrödinger or field representation $[2,3]$ in which the state vector $\psi=\psi(\mathscr{F})$ is a functional of the field configurations on a spacelike hyperplane with unit normal $n_{\mu}$. We choose a temporal gauge,

$$
A^{a}{ }_{\mu} n^{\mu}=0 .
$$

The fields $\mathscr{F}$ are assumed to have asymptotic behavior

$$
A_{i}^{a}=O\left(r^{-1}\right), \sigma^{I}=O\left(r^{-1}\right),|\phi|-1=O\left(r^{-1}\right),
$$

and the space of such $\mathscr{F}$ consists of disjoint sectors labelled by the (integral) values of the Euler index

$$
m=\frac{1}{8 \pi} \oint \varepsilon_{a b c} \Phi^{a} \partial_{i} \Phi^{b} \partial_{j} \Phi^{c} \varepsilon^{i j k} d S_{k}
$$

where the integral is over any sphere enclosing all the zeros of $\Phi$. We further restrict the gauge so that in each sector, 


$$
\left.\begin{array}{l}
A^{a}{ }_{i}=\varepsilon^{a b c} \partial_{i} \Phi_{b} \Phi_{c}+O\left(\frac{1}{r^{2}}\right), \\
\Phi^{a}=\hat{x}^{a} \sin \theta \cos m \phi+\hat{y}^{a} \sin \theta \sin m \phi+\hat{z}^{a} \cos \theta+O\left(\frac{1}{r}\right), \\
\sigma^{I}=O\left(r^{-1}\right),
\end{array}\right\}
$$

where $\hat{x}^{a}, \hat{y}^{a}$ and $\hat{z}^{a}$ are an orthonormal basis of isovectors and $(r, \theta, \phi)$ are spherical coordinates in the physical space.

In a Higgs vacuum, a region where

$$
|\Phi|=1, D_{\mu} \Phi^{a}=0, \quad \text { and } \quad \sigma^{I}=0,
$$

one can define the electromagnetic field $F_{\mu \nu}$ by

$$
F_{\mu \nu}^{a}=e \Phi^{a} F_{\mu \nu} .
$$

The index $m$ is then related to the total magnetic charge $Q_{B}$ by

$$
Q_{B}=\frac{1}{4 \pi} \int_{\infty} B^{i} d S_{i}=-\frac{m}{e},
$$

where $B^{i}=\frac{1}{2} \varepsilon^{i j k} F_{j k}$, and the smallest unit of electric charge will be $\frac{e}{2}$.

In the Schrödinger representation the field operators $\underset{\sim}{A}{ }_{i}^{a}(x), \underset{\sim}{\Phi}(x),{\underset{\sim}{i}}^{i}(x)$ have the meaning

$$
\begin{aligned}
&{\underset{\sim}{A}}_{i}^{a}(x) \psi(\mathscr{F})= A_{i}^{a}(x) \psi(\mathscr{F}), \Phi^{a}(x) \psi(\mathscr{F})=\Phi^{a}(x) \psi(\mathscr{F}), \\
& \sigma^{I}(x) \psi(\mathscr{F})=\sigma^{I}(x) \psi(\mathscr{F}),
\end{aligned}
$$

while the corresponding momentum operators (operator valued distributions) are

$$
\prod_{\sim}^{i}(x)=\frac{1}{i} \frac{\delta}{\delta A_{i}^{a}(x)}, \prod_{\sim}(x)=\frac{1}{i} \frac{\delta}{\delta \Phi^{a}(x)}, \prod_{\sim}(x)=\frac{1}{i} \frac{\delta}{\delta \sigma^{I}(x)},
$$

where, for example,

$$
\Pi_{i}^{a}=\frac{\partial \mathscr{L}}{\partial A_{a}^{i}}=\frac{1}{4 \pi e^{2}} F_{0 i}^{a}-\frac{\theta}{8 \pi^{2}} *^{a}{ }_{0 i} .
$$

The state vectors $\psi$ are governed by the Gauss constraint equation

$$
\left[D_{i} \prod_{\sim}^{i}+\varepsilon_{a b c} \Phi_{\sim}^{b} \prod_{\sim}^{c}-\frac{i}{2} \tau_{a}^{I}{ }_{J}\left(\sigma^{J} \prod_{\sim} \prod_{I}-\sigma_{I}^{\dagger} \prod_{\sim}^{\dagger J}\right)\right] \psi=0,
$$

and by the equation of motion

$$
\underset{\sim}{H} \psi=i \partial_{t} \psi
$$

where $\underset{\sim}{H}$ is the Hamiltonian operator associated with the Lagrangian (1). Only the constraint Eq. (16), however, will play a direct role in the discussion of statistics and spin. Its left hand side is a distribution, and the meaning of the equation is clear when one integrates it against a test function $\zeta^{a}$ of compact support (i.e., 
vanishing outside a bounded region): after an integration by parts one has

$$
\begin{aligned}
0= & \int d^{3} x\left\{-D_{i} \zeta^{a}(x) \frac{\delta}{\delta A_{i}^{a}(x)}+\varepsilon_{b c}^{a} \zeta^{b}(x) \Phi^{c}(x) \frac{\delta}{\delta \Phi^{a}(x)}\right. \\
& \left.-\frac{i}{2} \tau_{a}{ }^{I} \zeta^{a}(x)\left(\sigma^{J}(x) \frac{\delta}{\delta \sigma^{I}(x)}-\sigma^{\dagger J}(x) \frac{\delta}{\delta \sigma^{\dagger I}(x)}\right)\right\} \psi \\
= & \left.\frac{d}{d \chi} \psi\left(A_{i}^{a}-\chi D_{i} \zeta^{a}, \Phi^{a}+\chi \varepsilon^{a}{ }_{b c} \zeta^{b} \Phi^{c}, \sigma^{I}-\frac{i}{2} \chi \tau_{a{ }^{I}}{ }^{I} \zeta^{a} \sigma^{J}\right)\right|_{\chi=0} .
\end{aligned}
$$

That is,

$$
\left.\frac{d}{d \chi} \psi(U(\chi) \mathscr{F})\right|_{\chi=0}=0
$$

where $U(\chi)$ denotes the gauge transformation (5), with

$$
U=\exp \left(\frac{i}{2} \chi \zeta^{a}(x) \tau_{a}\right)
$$

Replacing $\mathscr{F}$ by $\underset{\sim}{U}\left(\chi_{o}\right) \mathscr{F}$ in Eq. (19), we have

$$
\left.\frac{d}{d \chi} \psi(\underset{\sim}{U}(\chi) \mathscr{F})\right|_{\chi=\chi_{o}}=0 \text {, all } \chi_{o},
$$

whence $\psi(\underset{\sim}{U}(\chi) \mathscr{F})=\psi(\mathscr{F})$ for any member $\underset{\sim}{U}(\chi)$ of a family of asymptotically trivial gauge transformations for which $U(0)$ is the identity. That is, denoting by $\mathscr{G}_{o}^{\infty}$ the component of the identity in the group $\mathscr{G}^{\infty}$ of smooth asymptotically trivial gauge transformations, we have

$$
\psi(\underset{\sim}{\mathscr{F}})=\psi(\mathscr{F}), \text { all } U \text { in } \mathscr{G}_{o}^{\infty} .
$$

Equation (21) implies that two gauge transformations $U_{1}, U_{2}$ have the same action on the state space $\mathscr{H}:=\{\psi\}$ if they differ by an element $U_{o}$ of $\mathscr{G}_{0}^{\infty}$. In particular, the action of a gauge transformation that is asymptotically trivial but not in the component of the identity depends only on the winding number $k \in \mathscr{G}^{\infty} / \mathscr{G}_{0}^{\infty} \simeq \mathbb{Z}$; there is in each superselected sector an angle $\hat{\theta}$ [2] such that for all $U \in \mathscr{G}^{\infty}$,

$$
\underset{\sim}{\psi} \psi=e^{i k \hat{\theta}} \psi \text {. }
$$

As mentioned earlier, introducing the term $\frac{\theta}{32 \pi^{2}} F^{a}{ }_{\mu \nu}{ }^{*} F_{a}{ }^{\mu \nu}$ in the Lagrangian density allows one to set

$$
\hat{\theta}=0
$$

without loss of generality, and we will do this. Then $\mathscr{H}$ is invariant under the full group $\mathscr{G}^{\infty}$ of asymptotically trival gauge transformations.

Let $\mathscr{C}=\bigcup_{m} \mathscr{C}_{m}$ denote the space of equivalence classes of fields satisfying the asymptotic conditions (10), where

$$
\mathscr{F} \sim \mathscr{F}^{\prime} \Leftrightarrow \mathscr{F}^{\prime}=\bigcup \underset{\sim}{ } \mathscr{F}, \text { some } U \in \mathscr{G}^{\infty} .
$$


Then state vectors $\psi \in \mathscr{H}$ are functions on $\mathscr{C}$,

$$
\psi([\mathscr{F}]):=\psi(\mathscr{F}),
$$

where $[\mathscr{F}] \in \mathscr{C}$ is the equivalence class containing $\mathscr{F}$.

There is, however, a set of gauge transformations which, although not asymptotically trivial, leave invariant the asymptotic conditions (10). These have the asymptotic form of rotations of isovector space by a constant angle $\chi$ about the Higgs field. That is, $U(\chi)$ has the form (20) with

$$
\begin{gathered}
\zeta^{a}=\Phi^{a}+O\left(r^{-1}\right), \\
D_{i} \zeta^{a}=O\left(r^{-2}\right),
\end{gathered}
$$

and because two transformations that differ by an element of $\mathscr{G}^{\infty}$ have the same action on $\mathscr{C}$ and $\mathscr{H}$, the action $\underset{\sim}{U}$ depends only on the angle $\chi$ or, more precisely, on an element $u=e^{i \chi / 2}$ of the group $U(1)$.

We denoted by $U$ the action on $\mathscr{H}$ or $\mathscr{C}$ of $U \in \mathrm{SU}(2)$. Similarly, we denote by $\underset{\sim}{\mathcal{A}}$ or $u(\chi)$ the action on $\mathscr{H}$ or $\mathscr{C}$ of an element $u=e^{i \chi} \in \mathrm{U}(1)$, given by

$$
\underset{\sim}{u}(\chi) \psi:=U(2 \chi) \psi, \quad \underset{\sim}{u}(\chi)[\mathscr{F}]:=\underset{\sim}{U}(2 \chi)[\mathscr{F}],
$$

where

$$
U(2 \chi)=\exp \left(i \chi \zeta^{a} \tau_{a}\right)
$$

with $\zeta^{a}$ satisfying (25). Formally, if $\mathscr{G}$ is the group of all gauge transformations that preserve the asymptotic conditions (10), then $\mathscr{G} / \mathscr{G}^{\infty} \simeq \mathrm{U}(1)$, and the isomorphism is

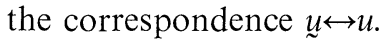

The action of this electromagnetic $\mathrm{U}(1)$ is related to the total electric and magnetic charge by the Gauss constraint (16). When the vector $\zeta^{a}$ in Eq. (18) has the asymptotic form (25), the integration by parts leading to (19) leaves as a surface integral a linear combination $q[6]$ of the electric and magnetic charge operators $Q_{E}$ and $Q_{B}$ :

$$
\underset{\sim}{q} \psi:=\left(\underset{\sim}{Q_{E}}+\frac{e^{2} \theta}{2 \pi}{\underset{\sim}{B}}_{B}\right) \psi=\frac{1}{4 \pi e} \int_{\infty} d S_{i} \Phi^{a}\left(F_{a}{ }^{0 i}-\frac{e^{2} \theta}{2 \pi} * F_{a}{ }^{0 i}\right) \psi=\left.\frac{e}{2 i} \frac{d}{d \chi} \underset{\sim}{u}(\chi) \psi\right|_{\chi=0} .
$$

Hence

$$
\underset{\sim}{u}(\chi)=\exp \left(\frac{2 i \chi}{e} \underset{\sim}{q}\right)
$$

Now $\underset{\sim}{u}(2 \pi)=1$ because it is asymptotically trivial, from which follows the charge quantization condition

$$
q=\frac{n}{2} e
$$

(When $\hat{\theta} \neq 0, \mathscr{H}$ is invariant only under gauge transformations $U \in \mathscr{G}_{o}^{\infty}$. State vectors are functions on classes $\hat{\mathscr{C}}$ of fields equivalent with respect to $\mathscr{G}_{o}^{\infty}$ and the 
larger group $\mathscr{G} / \mathscr{G}_{0}^{\infty} \simeq \mathbb{R}$ acts nontrivially on $\hat{\mathscr{C}}$. The transformation $\underset{\sim}{\mathcal{u}}(2 \pi) \in \mathscr{G}^{\infty}$ has winding number $2 m$, whence on $\mathscr{H}_{m n}, u(2 \pi)=\exp \left(-2 i e \hat{\theta} Q_{B}\right)$, and Eq. (29) is replaced by $\hat{q} \equiv Q_{E}+\frac{e^{2}}{2 \pi}(\theta+\hat{\theta}) Q_{B}=\frac{n}{2} e$.)

The action $y(\pi)$ on $\mathscr{C}$ is equivalent (since equal asymptotically) to the action of $-1 \in \mathrm{SU}(2)$, namely,

$$
A^{a}{ }_{i} \rightarrow A_{i}^{a}, \Phi^{a} \rightarrow \Phi^{a}, \sigma^{I} \rightarrow-\sigma^{I} .
$$

In the absence of an isospinor field $\sigma,-1 \in \mathrm{SU}(2)$ acts as the identity on $\mathscr{C}$; thus the gauge group is in effect $\mathrm{SO}(3)=\mathrm{SU}(2) / \mathbb{Z}_{2}$, and only integral multiples of $e$ occur as eigenvalues of $q$. When $\psi$ has support on field configurations with nonzero $\sigma$, halfintegral multiples of $e$ occur as well. These half-integral eigenvalues are related to the occurrence of states with half-integral angular momentum [7].

b. Spin from Isospin. One can now define momentum and angular momentum operators on the state space $\mathscr{H}$ via the action of rotations and translations on $\mathscr{C}$. Let $\xi^{i}$ be a rotational or translational symmetry vector; that is, for a unit vector $n^{i}$,

$$
\xi_{\text {rot }}^{i}=\varepsilon^{i}{ }_{j k} r^{j} n^{k}
$$

generates rotations about the $n$-axis, while

$$
\xi_{\text {transl }}^{i}=n^{i}
$$

generates translations along $n^{i}$. In the rotational case let $R(\eta n)_{j}^{i}:=\exp (\eta \xi)_{j}^{i}$ be the rotation of $\mathbb{R}^{3}$ by an angle $\eta$ about the $n$-axis. Denote by $R(\eta n)[\mathscr{F}]$ the associated action on $[\mathscr{F}] \in \mathscr{C}$; by $T(\eta n)[\mathscr{F}]$ the action of a translation by $\eta n$ on $[\mathscr{F}]$; and by $\delta[\mathscr{F}]$ or $\delta_{\xi}[\mathscr{F}]$ the corresponding infinitesimal change in $[\mathscr{F}]$ : for example:

$$
\delta_{\xi_{\mathrm{rot}}}[\mathscr{F}]:=\left.\frac{d}{d \eta} \underset{\sim}{R}(\eta n)[\mathscr{F}]\right|_{\eta=0} .
$$

A realization $(\underset{\sim}{R}, \underset{\sim}{T})$ on $\mathscr{C}_{m}$ of the (covering group $\overline{\mathscr{E}}$ of) the euclidean group $\mathscr{E}$ can be found satisfying

(i) $\left[\delta_{\xi}, \delta_{\zeta}\right][\mathscr{F}]=\delta_{[\xi, \zeta]}[\mathscr{F}]$;

(ii) to within a gauge transformation, $A_{i}^{a}, \Phi^{a}$ and $\sigma^{I}$ behave like a spatial vector and two spatial scalars, respectively;

(iii) the asymptotic conditions (10) are preserved.

Simply rotating $A_{i}^{a}, \Phi^{a}$ and $\sigma^{I}$ as a spatial scalar and two spatial vectors will change the asymptotic behavior of $\Phi^{a}$ and $A_{i}^{a}$ unless the rotation is accompanied by a gauge transformation. If $m$ is odd, the gauge transformation needed to preserve the asymptotics makes the realization of $\mathrm{SO}(3)$ double-valued when the isospinor field $\sigma^{I}$ is nonzero [3].

The infinitesimal rotation $\delta_{\xi}[\mathscr{F}]$ satisfying (i)-(iii) is, in the notation of Eqs. (6) and (7),

$$
\delta_{\xi}=-\xi^{j} \mathscr{D}_{j}-\Delta\left(\zeta^{a}\right)
$$

where

$$
\zeta^{a}=m \hat{r} \cdot n \Phi^{a}+O\left(r^{-1}\right)
$$


Explicitly

$$
\begin{gathered}
\delta_{\xi} \Phi^{a}=-\xi^{j} D_{j} \Phi^{a}, \\
\delta_{\xi} A^{a}{ }_{i}=-\xi^{j} F^{a}{ }_{j i}-D_{i} \zeta^{a}, \\
\delta_{\xi} \sigma^{I}=-\xi^{j} D_{j} \sigma^{I}-\frac{i}{2} \zeta^{a} \tau_{a}{ }_{a}{ }_{J} \sigma^{J} .
\end{gathered}
$$

Infinitesimal translation by $n^{i}$ is given by ${ }^{1}$

$$
\delta_{n}=-n^{j} \mathscr{D}_{j} .
$$

To see when the realization is double-valued, it suffices to look at rotations about the $z$-axis. Consider $\mathscr{C}_{m}$ (the subspace of $\mathscr{C}$ for which the Euler index (9) has the value $m$ ).

For $n^{i}=\hat{z}^{i}$, Eqs. (32) and (7b) give

$$
\delta_{\xi}=-£_{\xi}+\Delta\left(A^{a}{ }_{j} \xi^{j}-\zeta^{a}\right) .
$$

By Eq. (10), $A^{a}{ }_{j}{ }^{j}-m \hat{r} \cdot \hat{n} \Phi^{a}=m \hat{z}^{a}+O\left(r^{-1}\right)$, whence

$$
\delta_{\xi}=-£_{\xi}+\Delta\left(f m \hat{z}^{a}\right),
$$

where $f$ is any function with asymptotic behavior $f=1+O\left(r^{-1}\right)$. For $f(r) \equiv 1$, the finite rotations corresponding to (34a) are

$$
R(\eta \hat{z})[\mathscr{F}]=\underset{\sim}{U}\left(\eta m \hat{z}^{a}\right)[\hat{R}(\eta \hat{z}) \mathscr{F}],
$$

where

$$
\hat{R} \mathscr{F}(r):=\left.\left(R_{i}^{j} A_{j}^{a}, \Phi^{a}, \sigma^{I}\right)\right|_{R^{-1}(r)}
$$

is the rotation of $\mathscr{F} \equiv\left(A^{a}{ }_{i}, \Phi^{a}, \sigma^{I}\right)$ as a vector and two scalars, and $U\left(\eta m \hat{z}^{a}\right)=\exp \left[\frac{i}{2} \eta m \hat{z}^{a} \tau_{a}\right] \in \operatorname{SU}(2)$. For $\eta=2 \pi, U\left(2 \pi m \hat{z}^{a}\right)=(-1)^{m}$, and by (30) we have $\underset{\sim}{R}(2 \pi)=\underset{\sim}{u}(m \pi)$. Thus on $\mathscr{H}$ we have from (28),

$$
\underset{\sim}{R}(2 \pi)=\underset{\sim}{u}(m \pi)=\exp \left(2 \pi i \underset{\sim}{\underset{\sim}{q}} Q_{B}\right),
$$

and a state $\psi$ is spinorial - is a superposition of states with purely half-integral angular momenta - precisely when it is an eigenstate for which $q Q_{B}=\frac{1}{2} m n$ is halfintegral. In the $\theta=0$ sector, this is of course identical to the criterion for halfintegral spin for the quantum mechanical system of point electric and magnetic charges.

Denote by $\mathscr{H}_{m}$ the subspace of $\mathscr{H}$ for which $\psi$ has support on $\mathscr{C}_{m}$. That is, $\mathscr{H}_{m}$ is the eigensubspace of $\mathscr{H}$ for which $Q_{B}$ has eigenvalue $-\frac{m}{e}$. Similarly, denote by $\mathscr{H}_{m n}$ the subspace of $\mathscr{H}_{m}$ for which $q$ has eigenvalue $n \frac{e}{2}$. Then $\underset{\sim}{R}(2 \pi)=(-1)^{m n}$ on $\mathscr{H}_{m n}$.

(When $\hat{\theta} \neq 0, \underset{\sim}{R}(2 \pi)$ differs from $\underset{\sim}{u}(m \pi)$ by a gauge transformation with winding number $m$. On $\mathscr{H}_{m n}, \underset{\sim}{R}(2 \pi)=e^{i \hat{\theta} m} \underset{\sim}{u}(\pi m)=\exp \left(2 \pi i \hat{q} Q_{B}\right)=(-1)^{m n}$.)

1 Eq. (33) is equivalent to the naive translation $\delta_{n} \mathscr{F}=-n^{j} \partial_{j} \mathscr{F}=-£_{n} \mathscr{F}$ because the difference, $\Delta\left(A_{j}^{a} n^{j}\right)$, generates a gauge transformation in $\mathscr{G}^{\infty}: A_{j}^{a} n^{j}=O\left(r^{-1}\right)$ 


\section{The $N$-Monopole Configuration Space}

a. $N$ Isolated Monopoles. To speak of particle exchange presupposes a notion of distinct particles. In a theory of solitons formed from an ordinary scalar field $\phi$, one could call two solitons distinct if they were separated by a region in which $\phi$ took on its vacuum value $\phi_{0}$, and state vectors having support on such configurations would then constitute the two soliton sector of the state space. In the Yang-Mills case, we will require that a single isolated monopole be surrounded by Higgs vacuum, Eq. (11). Then, from Eq. (12),

$$
F^{a}{ }_{i j}=e \Phi^{a} F_{i j} .
$$

In order to isolate the degrees of freedom proper to the monopoles from those corresponding to photons, we require further that in the Higgs vacuum $F_{i j}$ be the coulomb field of a magnetic charge, that is, the external field of the monopole in the low velocity approximation.

For $N$ distinct monopoles centered about points $o_{\alpha}, \alpha=1, \ldots, N$, the Higgs vacuum that separates them will again be characterized by Eq. (36) with $F_{i j}$ the sum of the magnetic coulomb fields $F_{i j}$ about each of the points $o_{\alpha}$ : If the monopole at $o_{\alpha}$ has magnetic charge $g_{\alpha}=-\frac{m_{\alpha}}{e}$, then

$$
F_{i j}(r)=\sum_{\alpha} \stackrel{\alpha}{F}_{i j}(r)=\sum_{\alpha} g_{\alpha} \frac{\varepsilon_{i j k} r_{\alpha}{ }^{k}}{\left|r_{\alpha}\right|^{3}},
$$

where $r_{\alpha}{ }^{i}:=r^{i}-o_{\alpha}{ }^{i}$. Formally, we define as follows the configuration space $\mathscr{C}^{N}$ of $N$ distinct monopoles of magnetic charges $g_{\alpha}{ }^{2}$.

Definition. $\mathscr{C}^{N}=\left\{[\mathscr{F}] \in \mathscr{C}\right.$ such that there is $R>0$ and $N$ disjoint balls $\beta_{\alpha}$ of radius $R$ and centers $o_{\alpha}$ for which $\}$;

(A) $\mathscr{F}$ is Higgs vacuum and satisfies (37) outside $\bigcup_{\alpha} \beta_{\alpha}$, where $g_{\alpha} \neq 0$; and

(B) $\Phi^{a}$ vanishes at each point $o_{\alpha}$ and nowhere else.

Condition (B) prevents one from classifying as a single monopole of magnetic charge $g$ a group of several monopoles having total magnetic charge $g$.

Similarly,

Definition. $\mathscr{C}_{m}^{N}=\left\{[\mathscr{F}] \in \mathscr{C}^{N}\right.$ such that $m_{\alpha}=m$, all $\left.\alpha\right\}$.

The corresponding state space of functionals $\psi$ with support on $\mathscr{C}^{N}$ will be written $\mathscr{H}^{N}$ (and similarly $\mathscr{H}_{m}^{N}$ corresponds to $\left.\mathscr{C}_{m}^{N}\right):^{3}$

2 In the Prasad-Sommerfield limit, the approximation of Higgs vacuum outside the monopoles is less appropriate because $\Phi^{a}$ fails to be covariantly constant in the same order that the coulomb field fails to vanish. For dynamical purposes, it would be better in this case to alter our definition of $\mathscr{C}^{N}$ to include the $1 / r$ contribution to the Higgs field of the monopoles. The resulting bundle, however is isomorphic to $\mathscr{C}^{N}$, because this $1 / r$ contribution to $\Phi^{a}$ is determined by the positions and magnetic charges of the monopoles

3 Two distinct idealizations are involved in treating $\mathscr{C}^{N}$ as the effective $N$-monopole configuration space. First, we are assuming a perfect Higgs vacuum separates the monopoles, and second we are ignoring the fact that an actual vacuum functional $\psi$ is only concentrated near, not actually on, the values of $\mathscr{F}$ described by (11), (36) and (37). These approximations become exact in the limit where both $\hbar$ and the monopole velocities vanish and where the heavy boson masses of the theory become infinite; and by continuity the statistics of the monopoles (the subject of paper II) are constant along the family of theories parameterized by $\hbar$ and $m$ 
Definition. $\mathscr{H}^{N}=\left\{\psi \in \mathscr{H}\right.$ such that $\psi(\mathscr{F})=0$ unless $\left.[\mathscr{F}] \in \mathscr{C}^{N}\right\}$.

Definition. For $\mathscr{F} \in \mathscr{C}^{N}$, the intermonopole region is the set $I:=\mathbb{R}^{3} \backslash \bigcup_{\alpha} \beta_{\alpha}$.

From the fact that the monopoles are surrounded by a Higgs vacuum it follows that there is an action of $U(1)$ on each monopole separately, in terms of which one can define the electric charge of individual monopoles. To see this, consider first a field configuration $\mathscr{F} \in \mathscr{C}^{1}$ describing a single monopole located at the origin $\left(o_{1}=0\right)$, and note that the gauge transformation $U(2 \chi)$ given by Eqs. (25), (26) is equivalent (equal modulo $\left.\mathscr{G}^{\infty}\right)$ to $\exp \left(i \chi f \Phi^{a} \tau_{a}\right)$, where $f$ is any function with $f=1$ outside some finite radius, say $r=R+\varepsilon$. Because a constant gauge rotation about $\Phi^{a}$ leaves $\Phi^{a}$ and $A^{a}{ }_{i}$ unchanged in the Higgs vacuum, we can, for the field $\mathscr{F}$ in question, replace (26) by

$$
\underset{\sim}{u}(\chi)=\underset{\sim}{U}
$$

where

$$
U= \begin{cases}\exp \left(i \chi f \Phi^{a} \tau_{a}\right), & r \leqq R+\varepsilon \\ 1, & r \geqq R+\varepsilon,\end{cases}
$$

without affecting $\underset{\sim}{\operatorname{u}} \mathscr{F}$. For example, if

$$
f(r)=\left\{\begin{array}{l}
0, \quad r \leqq R \\
\frac{1}{2}+\frac{1}{2} \tanh \left[\left(R+\frac{\varepsilon}{2}-r\right)(R+\varepsilon-r)^{-1}(R-r)^{-1}\right], \quad R \leqq r \leqq R+\varepsilon \\
1, \quad r \geqq R+\varepsilon,
\end{array}\right.
$$

then in the shell $R \leqq r \leqq R+\varepsilon, \underset{\sim}{u}$ is a gauge rotation about the $\hat{\Phi}^{a}$-axis by an angle that increases from 0 to $\chi$ as $r$ increases from $R$ to $R+\varepsilon$ (and $u \mathscr{F}=\mathscr{F}$ elsewhere) ${ }^{4}$.

The operator (38) will be called a $U(1)$ rotation. It is defined for any $\mathscr{F}$ which has Higgs vacuum in the shell $R<r<R+\varepsilon$, and for such an $\mathscr{F}$ can be described as a change in the "U(1)-orientation" of the interior region $r<R$ with respect to the exterior region $r>R$.

In an $N$-monopole configuration, one can choose $r$ to be the distance from the center of a particular monopole and take $\varepsilon$ small enough that no other monopole overlaps the region $r \leqq R+\varepsilon$. Then, once one has labelled (indexed) the $N$ monopoles of a field configuration $\mathscr{F}$, one acquires an action of $U(1) \times \ldots \times U(1)$ ( $N$-factors) on $[\mathscr{F}]$. Thus $\mathscr{C}^{N}$ can be viewed as a fiber bundle whose fibers are copies $^{5}$ of $U(1) \times \ldots \times U(1)$ and whose base space we will call $\mathscr{M}^{N}$ (i.e., $[\mathscr{F}]$,

4 Note that $\underset{\sim}{\mathcal{b}}(\chi)$ as given by (38) is not the gauge-transformation corresponding to the discontinuous function (38b) because it lacks the $\delta$-function term which would arise from a sudden drop of $\chi$ to zero at $r=R+\varepsilon$ (whence it can be equivalently described as the addition of a certain $\delta$-function term to the vector potential $A_{k}^{a}$ ). Except when $N=1$, it is thus not a gauge transformation at all, although it is everywhere locally equivalent to one in its effect on any $[\mathscr{F}] \in \mathscr{C}^{N}$. On the other hand, it will approximately commute with the Hamiltonian for as long as the monopoles remain (approximately) separated by Higgs vacuum, and is in that sense a dynamical symmetry of $\mathscr{C}^{N}$

5 Strictly speaking they are faithful copies only for configurations $\mathscr{F}$ such that $\sigma$ vanishes identically in no $\beta_{\alpha}$ 
$\left[\mathscr{F}^{\prime}\right] \in \mathscr{C}^{N}$ represent the same point of $\mathscr{M}^{N}$ if one can produce $\left[\mathscr{F}^{\prime}\right]$ from $[\mathscr{F}]$ by $\mathrm{U}(1)$-rotating each of the $N$-monopoles through an appropriate angle). We claim that a point of $\mathscr{M}^{N}$ represents the locations and internal configurations of the monopoles without specifying their phases.

More precisely, if $\mathscr{F} \in \mathscr{C}^{N}$ then we will call the centers $o_{\alpha}$ the locations of the $N$ monopoles and will define the internal configuration of the monopole at $o_{\alpha}$ by subtracting from $F^{a}{ }_{i j}$ a contribution representing the coulomb field of the other monopoles. In the Higgs vacuum this contribution is $\Phi^{a} F_{i j}^{\alpha}$, where

$$
\stackrel{\alpha}{F}_{i j}^{\mathrm{ext}}:=\sum_{\alpha^{\prime} \neq \alpha}{\stackrel{\alpha^{\prime}}{F}}_{i j}=F_{i j}-\stackrel{\alpha}{F}_{i j},
$$

while inside $\beta_{\alpha}$ there is no unique way to pick out an external field. In any event the monopole's statistics are unaffected by the choice and for simplicity we introduce a specific "external" vector potential $e \Phi^{a} A^{\alpha}{ }_{i}$, where

$$
A_{i}^{\alpha}{ }_{i}^{\mathrm{ext}}:=\int_{0}^{1} d t t r_{\alpha}^{j} F_{j i}^{\alpha}\left(t r_{\alpha}\right)
$$

is the unique vector potential for $\stackrel{\alpha}{F}^{\text {ext }}{ }_{i j}$ with $A_{i} r_{\alpha}{ }^{i}=0=A_{i}\left(r_{\alpha}=0\right)$. Let

$$
\mathscr{F}_{\alpha}^{\mathrm{int}}:=\left.\left(A^{a}{ }_{i}-e \Phi^{a} \stackrel{\alpha}{A}^{\mathrm{ext}}, \Phi^{a}, \sigma^{I}\right)\right|_{\beta_{\alpha}} .
$$

Definition. If $[\mathscr{F}] \in \mathscr{C}_{m}^{N}$, the $\alpha^{\text {th }}$ internal configuration of $[\mathscr{F}]$ is the equivalence class $[\mathscr{F}]_{\alpha}$, where $\mathscr{F} \sim \mathscr{F}^{\prime}$ iff $\mathscr{F}_{\alpha}^{\text {int }}$ and $\mathscr{F}_{\alpha}^{\prime \text { int }}$ are related by an arbitrary $\mathrm{SU}(2)$ gauge transformation on $\beta_{\alpha}$.

In particular, a $\mathrm{U}(1)$ rotation of the monopole at $o_{\alpha}$ reduces within $\beta_{\alpha}$ to a gauge transformation and therefore does not alter the internal configuration as just defined. Conversely, we have

Proposition. If $\left[\mathscr{F}^{\prime}\right],\left[\mathscr{F}^{\prime \prime}\right] \in \mathscr{C}_{m}^{N}$ have the same monopole locations $o_{1}, \ldots, o_{N}$ and the same internal configurations $[\mathscr{F}]_{1}, \ldots,[\mathscr{F}]_{N}$, then for some choice of phases $\chi_{1}, \ldots, \chi_{N},\left[\mathscr{F}^{\prime \prime}\right]={\underset{\sim}{u}}_{1}\left(\chi_{1}\right) \ldots{\underset{\sim}{N}}_{N}\left(\chi_{N}\right)\left[\mathscr{F}^{\prime}\right]$, where ${\underset{\sim}{u}}_{\alpha}(\chi)$ is the $\mathrm{U}(1)$ rotation by $\chi$ of the monopole at $o_{\alpha}$.

Proof. Convert $\mathscr{F}^{\prime \prime}$ to $\mathscr{F}^{\prime}$ as follows. (i) For each $\alpha$ carry out a gauge transformation in $\beta_{\alpha}$ to make $\left.\mathscr{F}^{\prime \prime}\right|_{\beta_{\alpha}}=\left.\mathscr{F}^{\prime}\right|_{\beta_{\alpha}}$. This is possible by the definition of "internal configuration" inasmuch as $\mathscr{F}^{\prime}$ and $\mathscr{F}^{\prime \prime}$ have the same external magnetic field in $\beta_{\alpha}$ (because the monopole locations are the same for both $\mathscr{F}^{\prime}$ and $\mathscr{F}^{\prime \prime}$ ). (ii) Carry out in $I$ a gauge transformation that makes $\left.\Phi^{\prime \prime}\right|_{I}=\left.\Phi^{\prime}\right|_{I}$. This is possible because if

$$
B(x):=\left\{\mathrm{U}(x) \in \mathrm{SU}(2) \mid \mathrm{U}(x): \Phi^{\prime \prime}(x) \mapsto \Phi^{\prime}(x)\right\}, \text { then } B:=\bigcup_{\mathrm{x}} B(x) \text { is a } \mathrm{U}(1)
$$

bundle over $I$ which admits a cross section inasmuch as it is trivial on each $\partial \beta_{\alpha}$ (the sphere of radius $R$ about $o_{\alpha}$ ). So far we have arranged $\sigma^{\prime \prime}=\sigma^{\prime}$ and $\Phi^{\prime \prime}=\Phi^{\prime}$ everywhere and $A^{\prime \prime}=A^{\prime}$ within the $\beta_{\alpha^{\prime}}$. (iii) In $I$ the remaining gauge freedom amounts to an isospin rotation by $\chi(x)$ about $\Phi(x)$ at each $x$. Since $F_{i j}^{\prime \prime}=F_{i j}^{\prime}$, a local choice of $\chi(x)$ exists to convert $A^{\prime \prime}$ to $A^{\prime}$, and a global choice is possible because 
$\pi_{1}(I)$ vanishes. Choose $\chi(x)$ to make $\left.A^{\prime \prime}\right|_{I}=\left.A^{\prime}\right|_{I}$, and use the overall $\mathrm{U}(1)$ freedom in $\chi$ to arrange $\chi(\infty)=0$. (iv) At this stage $\mathscr{F}^{\prime \prime}=\mathscr{F}^{\prime}$ everywhere but the gauge transformation inside the $\beta_{\alpha}$ (step (i)) will in general not match that outside the $\beta_{\alpha}$ (steps (ii), (iii)). Rectify this by appropriate $\mathrm{U}(1)$ rotations of the $N$ monopoles, which is possible because $\mathscr{F}^{\prime \prime}$ is Higgs vacuum in some neighborhood of each $\partial \beta_{\alpha}$.

In summary, a point $[\mathscr{F}] \in \mathscr{C}^{N}$ is given by specifying for each monopole: an internal configuration $[\mathscr{F}]_{\alpha}$, a location $o_{\alpha}$, and a phase describing the $\mathrm{U}(1)$ orientation of the monopole with respect to its environment.

(When $\mathscr{C}$ is formed by regarding only the connected component $\mathscr{G}_{o}^{\infty} \mathrm{C}^{\mathscr{G}}$ as pure gauge, the fibers of $\hat{\mathscr{C}}^{N}$ are isomorphic to $\mathbb{R} \times[\mathrm{U}(1)]^{N-1}$.)

We will consider only states $\psi \in \mathscr{H}_{m}^{N}$ for which each monopole is in an eigenstate of $q$. That is, if $\mathscr{F} \in \mathscr{C}^{N}$ and $S$ is a sphere in the Higgs vacuum enclosing exactly one of the $N$ monopoles described by $\mathscr{F}$, then

$$
\frac{1}{4 \pi} \int_{S} d S_{i}\left(E_{\sim}^{i}+\frac{e^{2} \theta}{\pi} \mathbb{B}^{i}\right) \psi(\mathscr{F})=n \frac{e}{2} \psi(\mathscr{F}) .
$$

As in the one monopole case, Eq. (43) is equivalent to

$$
\psi(u \mathscr{F})=u^{n} \psi(\mathscr{F}),
$$

for any $\mathscr{F} \in \mathscr{C}_{m}^{N}$ and any $\mathrm{U}(1)$ rotation by $u$ of one of the monopoles of $\mathscr{F}$.

Definition. $\mathscr{H}_{m n}^{N}=\left\{\psi \in \mathscr{C}_{m}^{N}\right.$ satisfying Eq. (44) $\}$.

Any $\psi \in \mathscr{H}_{m n}^{N}$ is a function on the bundle $\mathscr{C}_{m}^{N}$ and such a function belongs to $\mathscr{H}_{m n}^{N}$ iff its restriction to any fiber has the form $\psi_{0} e^{i n\left(\chi_{1}+\ldots+\chi_{N}\right)}$, where $\chi_{1}, \ldots, \chi_{N}$ are $N$ phases parametrizing that fiber. By the term $d y o n$ we will mean a monopole in an eigenstate of $\underset{\sim}{q}$ and ${\underset{\sim}{B}}_{B}$, and state vectors $\psi \in \mathscr{H}_{m n}^{N}$ will be called $N$-dyon states.

b. Rotations and Translations. To make sense of a rotation or translation of one monopole that keeps the others fixed, one must decompose the field $\mathscr{F}$ into a part due only to that monopole and a part representing the magnetic coulomb fields of the others. The Higgs vacuum outside the monopoles is characterized completely by the electromagnetic $F_{i j}$ which is the sum of the magnetic coulomb fields of each monopole. Within a monopole, however, any such decomposition - e.g. that of Eqs. (41-42) is arbitrary for finite monopole separation. Nonetheless, the resulting ambiguity disappears as the monopole separations become large compared to the size of each monopole, because in that limit each monopole finds itself in a small and approximately constant external field.

If $l$ is the minimum distance between monopoles (and $R$ is their radius), then to second order in $\lambda=\frac{R}{l}$, the magnetic field in the intermonopole region $I$ near the $\alpha^{\text {th }}$ monopole consists of the coulomb field of the $\alpha^{\text {th }}$ monopole and a constant field $F_{i j}^{\text {ext }}\left(o_{\alpha}\right)[$ see Eq. (40)] from the other monopoles,

$$
F^{a}{ }_{i j}=e \Phi^{a}\left[\stackrel{\alpha}{F}_{i j}+F_{i j}^{\mathrm{ext}}\left(o_{\alpha}\right)(1+O(\lambda))\right]
$$

and

$$
F_{i j}^{\mathrm{ext}}\left(o_{\alpha}\right)=O\left(\lambda^{2}\right) \stackrel{\alpha}{F}_{i j} .
$$


A rotation of the monopole at $o_{\alpha}$ may be consistently defined to leading order in $\lambda$ by the prescription for an isolated monopole; that is, one may to lowest order ignore the external coulomb field entirely. In order to speak of the translation of a monopole across a distance comparable to $l$, however, it is necessary to keep the $O\left(\lambda^{2}\right)$ term $F_{i j}^{\text {ext }}\left(o_{\alpha}\right)$. This is not surprising; its meaning is, as we will see, that for $R \ll l$ the monopole behaves like a particle of charge $\frac{e}{2}$ in an external magnetic field. When translated about a closed loop it changes its phase by an amount proportional to the magnetic flux through the loop.

Translations in a constant external field. Because this key feature is already present for translations of one monopole in a constant external field, it will be helpful briefly to consider that case first. Let a monopole of radius $R$ and Euler index $m$ be centered at the origin, and suppose that in the Higgs vacuum (outside $R$ ), the Yang-Mills field has the form

$$
F_{i j}^{a}=e \Phi^{a}\left(F_{i j}^{\mathrm{int}}+F_{i j}^{\mathrm{ext}}\right),
$$

where

$$
F_{i j}^{\mathrm{int}}=-\frac{m}{e} \varepsilon_{i j k} \frac{r^{k}}{r^{3}}
$$

is the magnetic field of the monopole and $F^{\text {ext }}{ }_{i j}$ is a constant magnetic field. As in $\mathrm{Sect}$. IIb, we define translations by requiring that to within a gauge transformation $\mathscr{F}=\left(A_{i}^{a}, \Phi^{a}, \sigma^{I}\right)$ behave like a spatial vector and two spatial scalars and that the asymptotic form of the fields be preserved. Since $F^{\text {ext }}{ }_{i j}$ is constant it is translation invariant, and there is no need to subtract off an "external" part from $A_{i}^{a}$ before translating $\mathscr{F}$.

The present asymptotic behavior differs from that of Eq. (10) by the addition of the constant field $F^{\mathrm{ext}}{ }_{i j}$. For definiteness, we may choose the vector potential to have the asymptotic form

$$
A_{i}^{a}=\varepsilon_{b c}^{a} \partial_{i} \Phi^{b} \Phi^{c}+\Phi^{a} r^{k} e F_{k i}^{\mathrm{ext}}+O\left(\frac{1}{r}\right)
$$

but our prescription will depend only on the asymptotic form of $F^{a}{ }_{i j}$ and $\Phi^{a}$.

Let $h$ be the scalar

$$
h(x)=e n^{j} F_{j k}^{\mathrm{ext}} r^{k},
$$

chosen so that $\partial_{i} h=e n^{j} F_{j i}^{\mathrm{ext}}$. Translations may then be defined by writing

$$
\delta_{n}=-n^{j} \mathscr{D}_{j}+\Delta\left(h \Phi^{a}\right) .
$$

The change in the asymptotic form of $\mathscr{F}$ that would arise from $F^{\mathrm{ext}}{ }_{i j}$ had we used the form (33) is compensated in (50) by an infinitesimal gauge transformation generated by $h \Phi^{a}$. Outside the monopole, because $\sigma^{I}=0=D_{i} \Phi^{a}$, Eq. (50) becomes

$$
\left.\begin{array}{l}
\delta A_{i}^{a}=-e \Phi^{a} n^{j} F^{\mathrm{int}}{ }_{j i} \\
\delta \Phi^{a}=\delta \sigma^{I}=0
\end{array}\right\},
$$


whereby the asymptotic conditions are preserved. Thus apart from the term $\Delta\left(h \Phi^{a}\right),(50)$ agrees with the prescription for no external field. The meaning of this term can be seen from the commutator of two infinitesimal translations $n$ and $\bar{n}$, namely

$$
\left[\delta_{n}, \delta_{\bar{n}}\right]=\Delta\left[e F_{i j}^{\mathrm{ext}} n^{i} \bar{n}^{j} \Phi^{a}+O\left(\frac{1}{r}\right)\right] .
$$

That is, the result of translating a monopole in a closed loop of sides $n^{i}$ and $\bar{n}^{i}$ is a $\mathrm{U}(1)$ rotation of angle $\frac{e}{2} F^{\mathrm{ext}}{ }_{i j} n^{i} \bar{n}^{j}$, proportional to the flux through the loop. Hence for a state vector $\psi \in \mathscr{H}_{m n}$ of charge $n \frac{e}{2}$, the resulting change of phase is just that corresponding to the translation of a charged particle in an external magnetic field $F^{\mathrm{ext}}{ }_{i j}$.

Rotations and translations in $\mathscr{C}_{m}^{N}$. We can now define infinitesimal rotations and translations of a single monopole of $\mathscr{C}_{m}^{N}$. Because the monopole at $o_{\alpha}$ is immersed in the coulomb fields of the remaining monopoles, translations of it will not commute and one can weaken condition (i) of Sect. IIb to about $o_{\alpha}$.

(ia') $\left[\stackrel{\alpha}{\delta}_{\xi}, \stackrel{\alpha}{\delta}_{\eta}\right][\mathscr{F}]=\stackrel{\alpha}{\delta}_{[\xi, \eta]}[\mathscr{F}]$, when $\xi$ or $\eta$ is a rotational symmetry vector However, one has the additional requirement that

$\left(\mathrm{ib}^{\prime}\right)$ translation of a single monopole by $\xi^{i}$ is equivalent to translation of [ $\left.\mathscr{F}\right]$ itself by $\xi^{i}$ together with the translation of all other monopoles by $-\xi^{i}$.

Because translations and rotations should affect only the field "due to the $\alpha^{\text {th }}$ monopole," one must modify (ii) to require that

(ii') apart from gauge and U(1) phase, the fields $\mathscr{F}_{\alpha}^{\text {int }}=\left(\stackrel{\alpha}{A_{i}^{\text {int }, a}}, \Phi^{a}, \sigma^{I}\right)$ behave in $\beta_{\alpha}$ like a spatial scalar and two spatial vectors, respectively; in $I$ the contribution $\stackrel{\alpha}{F}_{i j}$ to the coulomb field arising from a translated monopole is the translated coulomb field; and in $\beta_{\alpha^{\prime}}, \alpha^{\prime} \neq \alpha,[\mathscr{F}]_{\alpha^{\prime}}$ is unchanged.

Finally,

(iii') the transformed configuration is again in $\mathscr{C}_{m}^{N}$.

An infinitesimal translation of the $\alpha^{\text {th }}$ monopole is then given as follows, with $h_{\alpha}=n^{j}\left(r-o_{\alpha}\right)^{k} e F_{j k}^{\mathrm{ext}}=n^{j}\left(r-o_{\alpha}\right)^{k} \sum_{\alpha^{\prime}} e F_{j k}^{\alpha}\left(o_{\alpha}\right)$; to leading order in $\lambda$

$$
\begin{aligned}
& \stackrel{\alpha}{\delta}_{n}=-n^{j} \mathscr{D}_{j}+\Delta\left(h_{\alpha} \Phi^{a}\right), \quad r \in \beta_{\alpha} ; \\
& \left.\begin{array}{l}
\stackrel{\alpha}{\delta}_{n} A_{i}^{a}=-e \Phi^{a} n^{j} F_{j i}^{\alpha} \\
\delta_{n} \Phi=\delta_{n} \sigma=0
\end{array}\right\} \quad r \in I ; \\
& \stackrel{\alpha}{\delta}_{n}=-\Delta\left[n^{j} e \stackrel{\alpha}{F}_{j k}\left(o_{\alpha^{\prime}}\right)\left(r-o_{\alpha^{\prime}}\right)^{k} \Phi^{a}\right], \quad r \in \beta_{\alpha^{\prime}}, \alpha^{\prime} \neq \alpha .
\end{aligned}
$$

Equations (53a-b) are analogous to Eqs. (50-51), while the gauge transformation of $(53 \mathrm{c})$ is needed to satisfy $\left(\mathrm{ib}^{\prime}\right)$. 
The commutator of two translations has the form of infinitesimal U(1) rotations of the monopoles. Writing $\mathscr{U}_{\alpha}(\chi)$ to denote the infinitesimal $\mathrm{U}(1)$ rotation of the monopole at $o_{\alpha}$

$$
\mathscr{U}_{\alpha}(\chi):= \begin{cases}\Delta\left(\Phi^{a} \chi\right), & r \in \beta_{\alpha} \\ 0, & r \notin \beta_{\alpha},\end{cases}
$$

we find

$$
\left[\stackrel{\alpha}{\delta}, \stackrel{\alpha}{\delta^{\delta}}\right]=-\mathscr{U}_{\alpha}\left(\sum_{\alpha^{\prime}}{\stackrel{\alpha^{\prime}}{F}}_{j k}\left(o_{\alpha}\right) n^{j} \bar{n}^{k}\right)-\sum_{\alpha^{\prime}} \mathscr{U}_{\alpha^{\prime}}\left(\stackrel{\alpha}{F}_{j k}\left(o_{\alpha^{\prime}}\right) n^{j} \bar{n}^{k}\right)
$$

Moreover, overall translation of $\mathscr{F}$ commutes with individual monopole translation,

$$
\left[\delta_{n}, \stackrel{\alpha}{\delta}_{\bar{n}}\right]=0 .
$$

Infinitesimal rotations of the $\alpha^{\text {th }}$ monopole about its center have, to leading order in $\lambda$, the form

$$
\stackrel{\alpha}{\delta}_{\xi}= \begin{cases}-\xi^{j} \mathscr{D}_{j}-\Delta\left(m \hat{r}_{\alpha} \cdot n \Phi^{a}\right), & r \in \beta_{\alpha} \\ 0, & r \notin \beta_{\alpha},\end{cases}
$$

where $\hat{r}_{\alpha}=\vec{r}_{\alpha} / r_{\alpha}=\left(\vec{r}-o_{\alpha}\right) /\left|\vec{r}-\vec{o}_{\alpha}\right|$ and $\xi^{i}=\varepsilon^{i}{ }_{j k} n^{j} r_{\alpha}{ }^{k}$ generates rotations about the $n$-axis through $o_{\alpha}$.

The translations and rotations defined by Eqs. (53) and (55) commute with the $U(1)$ transformations (38) of individual monopoles, and so respect the structure of the bundle $\mathscr{C}_{m}^{N}$.

Finite rotations generated by $\delta_{\xi}$ of $(55)$ can be cast into a form analogous to (34b). If, as before, $f(r)$ is a function with $f(r)=1$ for $r \geqq R+\varepsilon$, then $\Delta\left[\left(f\left(r_{\alpha}\right)-1\right) \zeta^{a}\right]$ generates gauge transformations in $\mathscr{G}^{\infty}$; if $\zeta^{a}=A^{a}{ }_{j} \xi^{j}-m \hat{r}_{\alpha} \cdot n \Phi^{a}$, then adding $\Delta\left[\left(f\left(r_{\alpha}\right)-1\right) \zeta^{a}\right]$ to Eq. (55) and using (7b) we find

$$
\stackrel{\alpha}{\delta}_{\xi}= \begin{cases}-£_{\xi}+\Delta\left[f\left(r_{\alpha}\right)\left(A_{j}^{a} \xi^{j}-m \hat{r}_{\alpha} \cdot n \Phi^{a}\right)\right], & r_{\alpha} \leqq R+\varepsilon \\ 0, & r_{\alpha} \geqq R+\varepsilon .\end{cases}
$$

For the gauge choice

$$
\left.\begin{array}{l}
\Phi^{a}=\hat{x}^{a} \sin \theta_{\alpha} \cos m \phi_{\alpha}+\hat{y}^{a} \sin \theta_{\alpha} \sin m \phi_{\alpha}+\hat{z}^{a} \sin \theta_{\alpha} \\
A^{a}{ }_{i}=\varepsilon_{b c}^{a}{ }_{b i} \Phi^{b} \Phi^{c}(1+O(\lambda)),
\end{array}\right\} R \leqq r_{\alpha} \leqq R+\varepsilon,
$$

$A^{a}{ }_{j} \xi^{j}-m \hat{r}_{\alpha} \cdot n \Phi^{a}=m \hat{z}^{a}$ in that shell.

Then

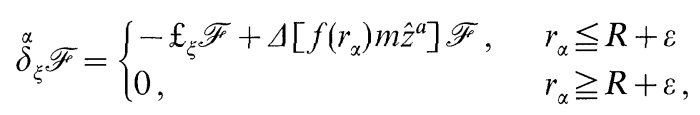

and the corresponding finite rotations of $\beta_{\alpha}$ by an angle $\eta$ are

$$
R_{\alpha}(\eta n)= \begin{cases}\underset{\mathscr{U}}{U}\left[f\left(r_{\alpha}\right) \eta \hat{z}^{a}\right] \hat{R}(\eta n) \mathscr{F}, & r_{\alpha} \leqq R+\varepsilon \\ \mathscr{F}, & r_{\alpha} \geqq R+\varepsilon .\end{cases}
$$




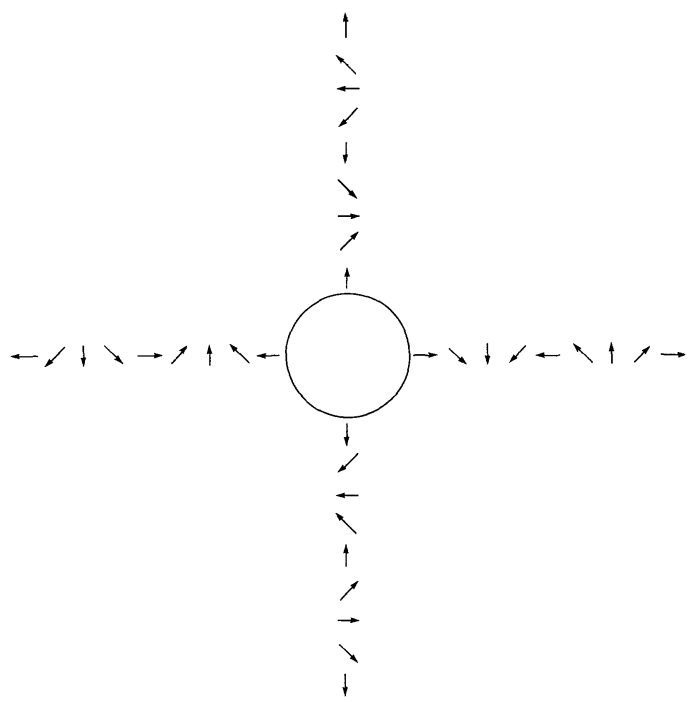

Fig. 1

For $f(r) \equiv 1, n^{i}=\hat{z}^{i}, R_{\alpha}$ coincides with Eq. (34b) applied to $\mathscr{C}_{m}^{1}$. For $f(r)$ given by Eq. (39), $\mathbb{\sim}_{\alpha}(2 \pi) \mathscr{F}$ is a gauge rotation of $\mathscr{F}$ about the $\hat{z}^{a}$ isospin axis by an angle that increases from 0 to $2 \pi$ as $r$ increases from $R$ to $R+\varepsilon$. Its effect on the Higgs field of an $m=1$ monopole is shown in Fig. 1.

An exact infinitesimal translation that agrees with Eqs. (53) is given as follows. Let

$$
h_{\alpha}=\int_{0}^{1} d t e n^{j} F_{j k}^{\mathrm{ext}}\left(t r_{\alpha}+o_{\alpha}\right) r_{\alpha}{ }^{k}=\sum_{\alpha^{\prime} \neq \alpha} h_{\alpha}^{\alpha^{\prime}},
$$

where

$$
h_{\alpha}^{\alpha^{\prime}}=\int_{0}^{1} d t e n^{j}{\stackrel{\alpha^{\prime}}{F}}_{j k}\left(t r_{\alpha}+o_{\alpha}\right) r_{\alpha}{ }^{k}
$$

Then

$$
\begin{aligned}
& \left.\begin{array}{l}
\stackrel{\alpha}{\delta}_{n} A^{a}{ }_{i}=-n^{j}\left(F_{j i}^{a}-e \Phi^{a} F_{j i}^{\mathrm{ext}}\right)-h_{\alpha} D_{i} \Phi^{a} \\
\stackrel{\alpha}{\delta}_{n} \Phi^{a}=-n^{j} D_{j} \Phi^{a} \\
\stackrel{\alpha}{\delta}_{n} \sigma^{I}=-n^{j} D_{j} \sigma^{I}-\frac{i}{2} h_{\alpha} \Phi^{a} \tau_{a}{ }^{I}{ }_{J} \sigma^{J}
\end{array}\right\}, \quad r \in I \cup \beta_{\alpha} \\
& \left.\begin{array}{l}
\stackrel{\alpha}{\delta}_{n} A^{a}{ }_{i}=-e \Phi^{a} n^{j} \stackrel{\alpha}{F}_{j i}+h_{\alpha^{\prime}}^{\alpha} D_{i} \Phi^{a} \\
\stackrel{\alpha}{\delta}_{n} \Phi^{a}=0 \\
\stackrel{\alpha}{\delta}_{n} \sigma^{I}=\frac{i}{2} h_{\alpha^{\prime}}{ }^{\alpha} \Phi^{a} \tau_{a J}{ }^{I} \sigma^{J}
\end{array}\right\}, \quad r \in \beta_{\alpha^{\prime}} \alpha^{\prime} \neq \alpha .
\end{aligned}
$$


The commutation relations (54) are exact for this law of parallel transport Exact infinitesimal rotations agreeing with Eqs. (55) are given by

$\stackrel{\alpha}{\delta}_{\xi} A^{a}{ }_{i}=-\xi^{j}\left(F^{a}{ }_{j i}-e \Phi^{a} F_{j i}^{\mathrm{ext}}\right)-\xi^{j} e A^{\mathrm{ext}} D_{i} \Phi^{a}-D_{i}\left(m \hat{r}_{\alpha} \cdot n \Phi^{a}\right)$

$\stackrel{\alpha}{\delta}_{\xi} \Phi^{a}=-\xi^{j} D_{j} \Phi^{a}$

$\stackrel{\alpha}{\delta}_{\xi} \sigma^{I}=-\xi^{j} D_{j} \sigma^{I}-\frac{i}{2}\left(\xi^{j} e A_{j}^{\mathrm{ext}}+m \hat{r}_{\alpha} \cdot n\right) \Phi^{a} \tau_{a}{ }^{I}{ }^{J} \sigma^{J}$

$r \in \beta_{\alpha}$,

$\stackrel{\alpha}{\delta}_{\xi}=0, \quad$ elsewhere.

Conditions (i')-(iii') are satisfied exactly by the translations and rotations (58) and (59).

\section{References}

1. See, for example, Jackiw, R.: Quantum meaning of classical field theory. Rev. Mod. Phys. 49, 681 (1977)

Goddard, P., Olive, D.: Rep. Prog. Phys. 41, 1357 (1978), and references therein

2. Jackiw, R., Rebbi, C. : Vacuum periodicity in a Yang-Mills quantum theory. Phys. Rev. Lett. 37, 172 (1976)

3. Wadia, S.: Canonical quantization of nonabelian gauge theory in the Schrödinger picture: Applications to monopoles and instantons. Ph. D. Dissertation, City University, New York (1978)

4. Callan, C.G., Dashen, R.F., Gross, D.J.: The structure of the gauge theory vacuum. Phys. Lett. 63B, 334 (1976)

5. Jackiw, R.: Gauge-covariant conformal transformations. Phys. Rev. Lett. 41, 1635 (1978)

6. Witten, E.: Dyons of charge $e \theta / 2 \pi$. Phys. Lett. 86B, 283 (1979)

7. Jackiw, R., Rebbi, C.: Spin from isospin in a gauge theory. Phys. Rev. Lett. 36, 1116 (1976) Hasenfratz, P., 't Hooft, G.: Fermion-boson puzzle in a gauge theory. Phys. Rev. Lett. 36, 1119 (1976)

Communicated by S.-T. Yau

Received June 18, 1982; in revised form November 2, 1982 
